TITLE:

\title{
High-Efficiency InGaN/GaN Light Emitters Based on Nanophotonics and Plasmonics
}

\author{
$\operatorname{AUTHOR}(\mathrm{S})$ :
}

Okamoto, Koichi; Kawakami, Yoichi

\section{CITATION:}

Okamoto, Koichi ...[et al]. High-Efficiency InGaN/GaN Light Emitters Based on Nanophotonics and Plasmonics. IEEE JOURNAL OF SELECTED TOPICS IN QUANTUM ELECTRONICS 2009, 15(4): 1199-1209

ISSUE DATE:

2009-07

URL:

http://hdl.handle.net/2433/109797

\section{RIGHT:}

(c) 2009 IEEE. Personal use of this material is permitted. However, permission to reprint/republish this material for advertising or promotional purposes or for creating new collective works for resale or redistribution to servers or lists, or to reuse any copyrighted component of this work in other works must be obtained from the IEEE. 


\title{
High-Efficiency InGaN/GaN Light Emitters Based on Nanophotonics and Plasmonics
}

\author{
Koichi Okamoto and Yoichi Kawakami
}

(Invited Paper)

\begin{abstract}
We report novel methods to enhance light emission efficiencies from InGaN/GaN quantum wells (QWs) based on nanophotonics and plasmonics. First, the nanoscopic optical properties were observed and characterized based on the carrier localization and the quantum confinement Stark effect depending on the In composition of InGaN. Based on the results, we proposed that the emission efficiencies should be improved by making nanostructures, and showed actual enhancement of photoluminescence (PL) intensities by using fabricated random nanodisk and arrayed nanopillar structures. Moreover, surface plasmon (SP) coupling technique was used to enhance blue and green light emissions from InGaN/GaN QWs. We obtained a 14-fold increase in the PL intensity along with a 7-fold increase in the internal quantum efficiency (IQE) of light emission from InGaN/GaN when nanostructured Ag layers were deposited $10 \mathrm{~nm}$ above the QWs. The possible enhancement mechanism was discussed and reproduced by using the 3-D finite-difference time-domain simulations. Electron-hole pairs in InGaN QWs couple to electron oscillations at the metal surface and produce SPs instead of photons or phonons. This new path increases the spontaneous emission rate and the IQEs. The SP-emitter coupling technique would lead to superbright and highspeed solid-state light-emitting devices that offer realistic alternatives to conventional fluorescent light sources.
\end{abstract}

Index Terms-LEDs, light sources, nanotechnology, optical materials, optoelectronic devices, photoluminescence (PL), plasmons, quantum wells (QWs), semiconductor-metal interfaces, spontaneous emission.

\section{INTRODUCTION}

$\mathbf{R}$ ECENTLY, solid-state light-emitting devices have been developed and expected as new-generation light sources because of their advantages such as small size, light weight, long lifetime, easy operation, and energy-saving. Since 1993, InGaN quantum wells (QWs) based LEDs have been continuously improved and commercialized as light sources in the UV and visible spectral regions [1]. In 1996, white light LEDs, in which a blue LED is combined with yellow phosphors $\left[\left(\mathrm{Y}_{1-\mathrm{a}} \mathrm{Gd}_{\mathrm{a}}\right)_{3}-\left(\mathrm{Al}_{1-\mathrm{b}} \mathrm{Ga}_{\mathrm{b}}\right)_{5} \mathrm{O}_{12}, \mathrm{Ce}^{3+}(\mathrm{YAG})\right]$, have been developed [2] and offer a replacement for conventional incandescent and fluorescent light bulbs. However, these devices have not

Manuscript received December 15, 2008; revised January 26, 2009.

$\mathrm{K}$. Okamoto is with the Department of Electronic Science and Engineering, Kyoto University, Kyoto 615-8510, Japan, and also with the Precursory Research for Embryonic Science and Technology (PRESTO) Program, Japan Science and Technology Agency, Kawaguchi 332-0012, Japan (e-mail: k.okamoto@hy4.ecs.kyoto-u.ac.jp).

Y. Kawakami is with the Department of Electronic Science and Engineering, Kyoto University, Kyoto 615-8510, Japan (e-mail: kawakami@kuee.kyotou.ac.jp)

Digital Object Identifier 10.1109/JSTQE.2009.2021530 fulfilled their original promise as solid-state replacements for light bulbs as their light emission efficiencies have been limited. The most important requirement for competitive LEDs for solid-state lighting is improvement of their quantum efficiencies of light emissions.

InGaN covers the full visible spectral range by controlling the bandgap energies from $\mathrm{InN}(0.6 \mathrm{eV})$ to $\mathrm{GaN}(3.4 \mathrm{eV})$. At the blue spectrum region, the external quantum efficiency $\left(\eta_{\text {ext }}\right)$ of the LED has achieved $75.5 \%$ at room temperature in spite of high threading dislocation (TD) [3]. However, such a high efficiency is achieved only in the blue spectral region, and it rapidly decreases outside this spectral range. Because of this reason, high-efficiency InGaN LEDs with green, yellow, or red spectrum regions have not been achieved so far. This so-called green gap issue is also one of the serious problems preventing an alternative of LEDs to conventional light bulbs.

The $\eta_{\text {ext }}$ value of LED is given by a product of the light extraction efficiency $\left(C_{\text {ext }}\right)$ and the internal quantum efficiency (IQE: $\eta_{\text {int }}$ ), which, in turn, is determined by the ratio of the radiative $\left(k_{\mathrm{rad}}\right)$ and nonradiative $\left(k_{\mathrm{non}}\right)$ recombination rates of carriers. Often, $k_{\text {non }}$ is faster than $k_{\text {rad }}$ at room temperature, resulting in modest $\eta_{\text {int }}$. Therefore, there are three methods to increase $\eta_{\text {ext }}$ : increase $C_{\text {ext }}$, decrease $k_{\text {non }}$, or increase $k_{\text {rad }}$. Several reports have been published to improve $C_{\text {ext }}$ from InGaN LEDs by using the patterned sapphire substrates and mesh electrodes [4] or making the photonic bandgap structures [5]. Much more effort has been placed into reducing $k_{\text {non }}$ by growing higher quality crystals because $\eta_{\text {ext }}$ of LEDs usually depend on the TD densities. For example, the TD densities were reduced to $10^{6} \mathrm{~cm}^{-2}$ by using the epitaxial lateral overgrowth (ELO) technique [6]. However, $\eta_{\text {ext }}$ of LEDs grown on ELO-GaN with longer wavelength emissions were not so improved, while those with UV-blue emission are well enhanced [7]. There have been very few studies focusing on increasing $k_{\text {rad }}$, though that could prove to be most effective for development of high $\eta_{\text {ext }}$ light emitters. Controlling of the radiative recombination process of carriers is very difficult because it depends on many factors. In this paper, we describe our proposed new techniques for the enhancement of $k_{\text {rad }}$ based on nanophotonics and plasmonics.

\section{Characterization of OpticAl Properties}

In order to control carrier recombination dynamics and improve the emission efficiencies, we have to elucidate and understand the important factors that determine the optical properties of InGaN QWs. It is known that microscopic inhomogeneities of optical properties of InGaN active layers are very important 

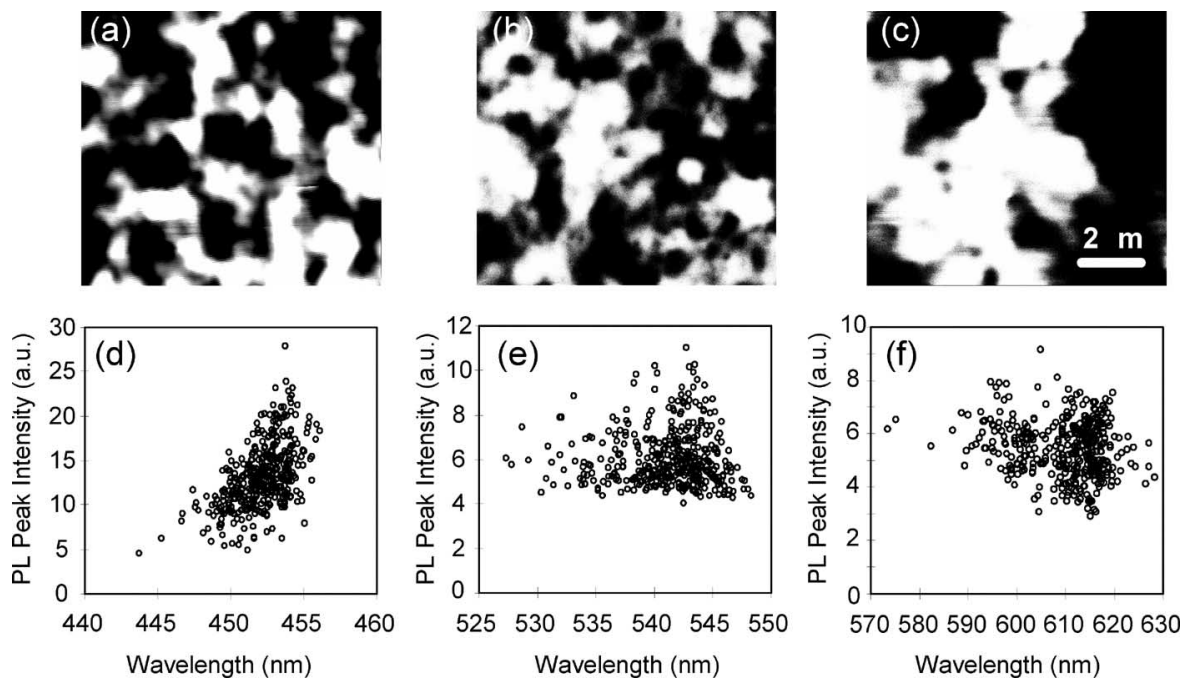

Fig. 1. CL images taken for InGaN/GaN QW that have (a) blue, (b) green, and (c) amber emissions at room temperature. Relationship between the PL peak intensities and wavelength of InGaN/GaN QW that have (d) blue, (e) green, and (f) amber emissions.

for emission efficiencies [8], [9]. The microscopic spatial inhomogeneities of the optical properties should be generated by a fluctuation of In composition by a phase separation in InGaN layers. The carriers are localized in small volumes of In-rich areas in $\mathrm{InGaN}$ and hindered to be trapped in nonradiative recombination centers. Another important feature of emission mechanics of InGaN/GaN is the quantum confinement Stark effect (QCSE) [10]. Strain in crystal induces piezoelectric fields in the energy band diagrams of InGaN separating electron-hole pairs and decreasing the emission efficiencies. The QCSE becomes more effective with increasing In composition in InGaN QWs. Avoiding QCSE is crucially important for higher performance of LEDs. For example, Walterelt et al. fabricated the piezoelectricfield-free GaN/AlGaN QW grown on $M$ plane of $\mathrm{GaN}$ substrate and observed about ten times faster spontaneous emission rate [11]. High-efficiency light emission from $\mathrm{InGaN} / \mathrm{GaN}$ QW grown on $\{11-22\}$ semipolar GaN substrate was also reported [12]. Local profiles of the QCSE due to the spatial inhomogeneity of the In composition are also expected to be a very important factor of the optical properties. Therefore, characterizations of nanoscopic optical properties should be necessary to understand the carrier dynamics and improve the emission efficiency.

Cathodoluminescence (CL) [13], [14], confocal scanning laser microscope (CSLM) [15], [16], and scanning near-field optical microscopy (SNOM) [17]-[19] are powerful methods to obtain optical images of InGaN/GaN in submicrometer scale. Fig. 1 shows the typical CL images taken for InGaN/GaN QW with (a) blue, (b) green, and (c) amber emission at room temperature. InGaN/GaN QW samples, which have 3-nm-thick active layers, were grown by a metal-organic chemical vapor deposition (MOCVD) method on a (0001) C-face sapphire substrate. These CL images have fine island-like structures with submicrometer scales due to the spatial fluctuation of the In compositions in InGaN active layers. The size of the islandlike structures of blue emission [see Fig. 1(a)] is of the order of submicrometer scale, but it increases with increasing of In composition and becomes larger than $1 \mu \mathrm{m}$ for the InGaN with amber emission [see Fig. 1(c)]. Such size should be much larger than the diffusion length of carriers.

Fig. 1 (d)-(f) shows the relationship between the CL peak intensities and wavelength at 400 arbitrarily selected points in Fig. 1 (a)-(c), respectively. For InGaN/GaN with blue emission, a clear increase along the wavelength was obtained [see Fig. 1(d)]. This correlation has been well interpreted by using the carrier localization, which should be the main origin for high emission efficiency of blue emission. On the other hand, the correlation of InGaN with green emission looked normally distributed [see Fig. 1(e)], while that of amber emission seemed to be decreasing along the wavelength [see Fig. 1(f)]. The correlation of amber emission is quite opposite to that of blue emission. This may suggest that the lower energy regions in the active layer of InGaN with amber emission should not be as effective as the localization center of carriers. We observed similar tendency by using CSLM [16] and SNOM [19], and it should be related to the reduction of emission efficiencies of InGaN LEDs with high In composition.

Moreover, the QCSE becomes effective under the higher In composition. The QCSE reduces the electron-hole wave function overlap and emission intensities at In-rich regions. This is the reason for the reduction of the emission efficiencies of LEDs with longer emission wavelength, and can explain the opposing CL intensity peak correlations of InGaN with high In compositions. The different behaviors of localization and the QCSE effect of electron-hole pairs in InGaN active layers should be attributed to the different size of the spatial inhomogeneities of In compositions. Based on these results, we propose that controlled spatial inhomogeneity in $\mathrm{InGaN}$ active layer should be more effective than making the crystal quality higher by reducing the TD density in order to improve emission efficiency of InGaN LEDs with high In composition. For example, making nanostructure may be one of the promising methods to control the spatial inhomogeneity in InGaN and improve the emission efficiencies by increasing $k_{\text {rad }}$. In the next section, we show 

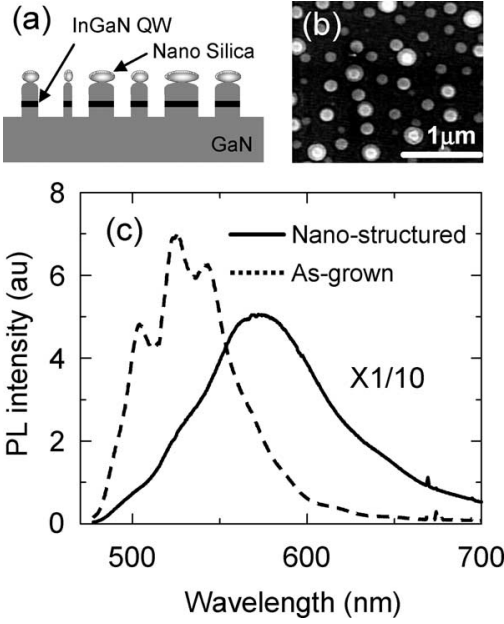

Fig. 2. (a) Sample structures of the fabricated nanodisks of InGaN/GaN QW by using dry etching process with nanosilica masks. (b) SEM image of the tip view of the sample. (c) PL spectra of the nanostructured and as-grown $\mathrm{InGaN} / \mathrm{GaN}$ at $10 \mathrm{~K}$.

the enhancements of photoluminescence (PL) of InGaN/GaN by making artificial nanodisks and nanopillar structures.

\section{NANOSTRUCTURE-ENHANCED Light EMISSIONS}

We fabricated nanodisk structures of InGaN by dry etching process with silica nanoparticles (20-200 nm diameters) dispersed on the sample surface as etching mask. The structure of the fabricated nanodisks was shown in Fig. 2(a). For the dry etching process, we used the chemically assisted ion beam etching (CAIBE). CAIBE is characterized by employing both chemical and physical etching processes, so that anisotropic but fast etching is possible under mild conditions [20]. In this study, Xe was used for sputtering, while $\mathrm{Cl}_{2}$ was used for chemical etching. The etched depth was $100 \mathrm{~nm}$. Fig. 2(b) shows the SEM image of the top view of the sample. The disk structures, which have submicrometer sizes, were observed at the random locations.

The PL spectra of the nanofabricated and the as-grown InGaN/GaN were shown in Fig. 2(c). To perform the PL measurements, a continuous-wave (CW) $\mathrm{He}-\mathrm{Cd}$ laser $(325 \mathrm{~nm})$ was used to excite the GaN cladding layers. PL was collected and focused into an optical fiber and subsequently detected with a multichannel spectrometer. The measurement was done at $10 \mathrm{~K}$ by using a cryocooler with helium compressor. The as-grown InGaN/GaN sample has green emission and the peak wavelength is around $520 \mathrm{~nm}$. On the other hand, the nanostructured sample has a very broad PL spectrum in the green-yellow wavelength region. This broad spectrum should be attributed to the nanodisk structures. The PL intensity from the nanodisk structures is about ten times stronger than that of as-grown sample. The origin of the intense emission should be due to the strong confinement of the electron-hole pairs into the nanodisk structures. This strong confinement should increase the electron-hole wave function overlap and radiative recombination rates, while the carrier localization is no longer effective in the as-grown sample as mentioned before. Moreover, the small-sized In-rich

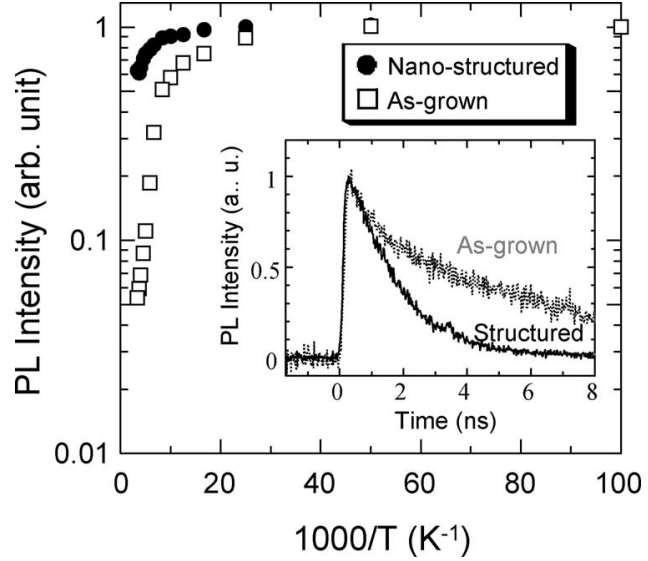

Fig. 3. Arrhenius plots of the integrated PL intensities of InGaN/GaN with random nanodisk structures and as-grown sample. PL integrated intensities at $10 \mathrm{~K}$ were normalized to 1 . (Inset) PL decay profiles of the same samples.

regions of the nanodisk structures may reduce the QCSE. The internal electric field should be reduced by the partial strain relaxation promoted by the size reduction. The enhancement of the carrier localization and the reduction of the QCSE increase the radiative recombination rates and the IQEs. The small structures can also enhance the light extraction efficiencies. The similar very broad PL spectra were observed when we used a diode laser (406 nm) to pump InGaN QW layer selectively. But in this case, the PL intensities of nanodisks were reduced compared to that of the as-grown sample because the absorption areas of $\mathrm{InGaN}$ became much smaller by making the nanodisk structures.

Fig. 3 shows Arrhenius plot of the integrated PL intensities of the nanostructured or the as-grown sample obtained by the temperature dependence measurements. The integrated PL intensities are constant in a low-temperature region, and then, decrease gradually with increasing temperature. The PL intensities of the as-grown sample become only $5 \%$ at room temperature. On the other hand, the PL intensities of nanodisks do not depend on temperature so much, and it has still $60 \%$ intensity at room temperature. The carrier dynamics in InGaN layer is well stabilized at a low temperature, and it is activated by thermal energy with increasing temperature and easily captured into the nonradiative recombination centers. In such a case, the IQE of emission can be estimated by assuming that $\eta_{\text {int }}$ is nearly equal to unity at low temperature $(10 \mathrm{~K})$. The IQE of InGaN with nanodisk structure was dramatically enhanced to $60 \%$, whereas the original value of unetched sample was $5 \%$. We achieved 12-fold improvement in $\eta_{\text {int }}$ of InGaN/GaN by making nanostructures. The inset of Fig. 3 shows the time profile of the PL probed by time-resolved PL (TRPL) measurements. To perform the TRPL measurements, the frequency-doubled output from a mode-locked Ti: $\mathrm{Al}_{2} \mathrm{O}_{3}$ laser was used to excite the InGaN. The pulsewidth, wavelength, and repetition rate were chosen as $1.5 \mathrm{ps}, 400 \mathrm{~nm}$, and $80 \mathrm{MHz}$, respectively. A streak camera served as the detector. It was found that the emission rate of the InGaN nanodisks is faster than that of the unetched sample. This should be attributed to the enhancement of the carrier localization and reduction of the QCSE by making nanodisk structures. 


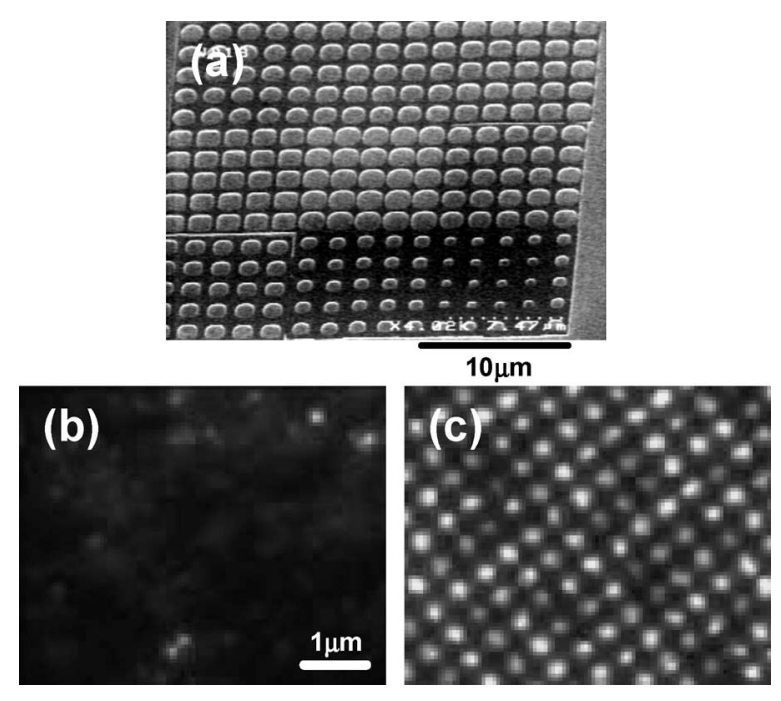

Fig. 4. (a) SEM image of the nanopillar array structures of InGaN/GaN fabricated by electron beam lithography and dry etching. Microscopic PL images of (b) nonstructured and (c) nanopillar array with $500 \mathrm{~nm}$ diameter.

We also fabricated the arrayed nanopillar structures by using the electron beam lithography. Fig. 4(a) shows the SEM images of the nanopillar arrays, which have several diameters. Such approach using lithography enables us to fabricate arbitrary structures at arbitrary positions. The patterns were defined into a 200-nm-thick polymethylmethacrylate (PMMA) resist spun onto the samples and exposed in a field emission electron beam lithography system. After lithography, the beam patterns were transferred into the samples by using the CAIBE. By this method, we succeeded to fabricate nanopillars whose smallest size was decreased to $50 \mathrm{~nm}$. Fig. 4 shows the microscopic PL images of (b) unpatterned and (c) nanostructured InGaN/GaN sample. Both images were taken in the same conditions and shown in same contrast, so it is clear that the emission intensity of the nanopillar arrays is much stronger than that of unpatterned sample. We also found that both the IQE and the emission rate of InGaN/GaN are increased by making the nanopillar array structures [21]. Moreover, we found an unexpected interesting phenomenon, which is the remarkable blinking of emission from the InGaN nanopillar arrays. This very impressive phenomenon of blinking has been reported for CdSe-based quantum dot structures [22], and is expected to be applicable to optical memory storage circuits and computing. We found that this is a very remarkable blinking phenomenon for InGaN/GaN nanopillars though this phenomenon was rarely reported for InGaN/GaN materials [23]. The nano sizes and arranged structures of lightemitting materials may enhance the blinking but the detail mechanism is still not clear. The details of this phenomenon would be reported elsewhere in future.

The similar enhancements of light emission feature have also been reported for the nanocolumn structures self-formed by molecular beam epitaxy [24]. From these nanostructures, we can control the carrier recombination dynamics and lightemission mechanism to improve the emission efficiencies. The optimized nanophotonics devices should be applied for use in high-efficiency LEDs, as well as in low-threshold lasers. How-

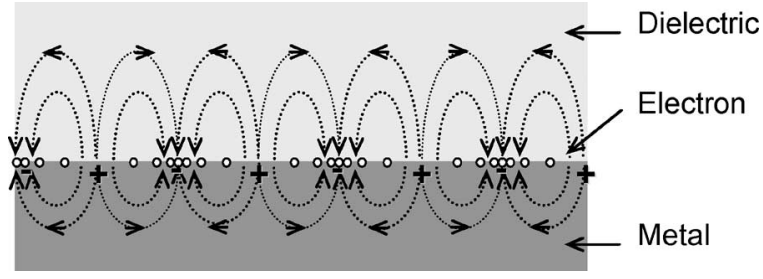

Fig. 5. Schematic diagram of the SP and SPP generated at the metal/dielectric interface.

ever, even if these nanostructures are used, superbright LEDs as alternative to conventional fluorescent light bulbs have not been achieved so far in spite of a lot of efforts. One problem may be due to the difficulty of the nanofabrication techniques. Other problem is the cost of LEDs, which are still much expensive than those of fluorescent light bulbs. Thus, the development of a new method, which must be much easier and more effective, has been earnestly desired for further improvements of emission efficiencies of InGaN LEDs. In the next section, we propose other method based on plasmonics as one solution to dramatically increase the efficiencies of light-emitting materials and devices.

\section{Surface-Plasmon-Enhanced Light EMissions}

\section{A. Applications of Plasmonics to Optics}

Conduction electron gas in a metal oscillates collectively, and the quantum of this plasma oscillation is called plasmon. A special plasma oscillation mode called surface plasmon (SP) exists at an interface between a metal, which has a negative dielectric constant, and a positive dielectric material [25]. The charge fluctuation of the oscillation of the SP is accompanied by fluctuations of electromagnetic fields, which is called SP polariton (SPP). Schematic diagrams of the SP and the SPP mode generated at metal/dielectric interface were shown in Fig. 5. The SPP can interact with light waves at the interface, and it renders novel optical properties and functions to materials. The technique controlling and utilizing the SPP is called "plasmonics" and has attracted much attention with the recent rapid advance of nanotechnology [26]-[28].

The wave vector of the SPP $\left(k_{\mathrm{SP}}\right)$ parallel to the interface can be written with the following equation when the relative permittivity of the metal is $\varepsilon_{1}=\varepsilon_{1}^{\prime}+\varepsilon_{1}^{\prime \prime} i$ and that of the dielectric material is $\varepsilon_{2}$ :

$$
k_{\mathrm{SP}}=\frac{\omega}{c} \sqrt{\frac{\varepsilon_{1}^{\prime} \varepsilon_{2}}{\varepsilon_{1}^{\prime}+\varepsilon_{2}}}+\frac{\omega}{c}\left(\frac{\varepsilon_{1}^{\prime} \varepsilon_{2}}{\varepsilon_{1}^{\prime}+\varepsilon_{2}}\right)^{3 / 2} \frac{\varepsilon^{\prime \prime}{ }_{1}}{2 \varepsilon_{1}^{\prime 2}} i
$$

where $\omega$ and $c$ are the frequency of the SPP and the light velocity in vacuum, respectively. The first and second terms of this equation give the dispersion and the damping factor of the SPP. The $k_{\mathrm{SP}}$ values are much larger than the wave vector of the light wave propagated in the dielectric media. This fact suggests that the SPP can propagate into nanospaces much smaller than the wavelength. This enables us to shrink the sizes of waveguides and optical circuits into nanoscale [27].

Wave vectors of the SPP perpendicular to the interface in a metal or a dielectric material must be an imaginary number 
because $k_{\mathrm{SP}}$ is larger than the light line. This suggests that the electromagnetic fields of the SPP are strongly localized at the interface, and it makes giant fields at the interface. This huge field enhancement effect is also one of the most important features of the SPPs. It has been applied to high sensitive sensors [28] using the surface-enhanced Raman scattering (SERS), SP resonance (SPR), and so on.

One futuristic application of plasmonics is the development of superbright LEDs. Since 1990, the idea of the SP-enhanced light emission was proposed and received much attention [29]-[32]. Vuckovic et al. reported about the SP-enhanced LED analyzing both theoretically and experimentally [30]. For InGaN QWs, Gontijo and coworkers reported the coupling of the spontaneous emission from QW into the SP on Ag thin films and showed increased absorption of light at the SP frequency [31]. Neogi et al. confirmed that the recombination rate in an $\mathrm{InGaN} / \mathrm{GaN}$ QW could be significantly enhanced by the TRPL measurement [32]. However, in these early studies for InGaN QWs, light could not be extracted efficiently from the $\mathrm{Ag} / \mathrm{GaN}$ surface. Therefore, the actual enhancements of visible light emissions had not been observed directly before our first report [33].

\section{B. Enhancement of PL of InGaN/GaN}

Recently, we have reported, for the first time, large PL increases from $\mathrm{InGaN} / \mathrm{GaN} \mathrm{QW}$ material coated with metal layers [33]. InGaN/GaN single-QW (3 nm) structures were grown by MOCVD, and $\mathrm{Ag}, \mathrm{Al}$, or Au layers $(50 \mathrm{~nm})$ were deposited on top of the surfaces of these wafers by a high vacuum thermal evaporation. The sample structure was shown in Fig. 6(a). To perform the PL measurements, a CW-InGaN diode laser $(406 \mathrm{~nm})$ was used to excite the QWs from the bottom surface of wafer. This backside access is the important trick that enabled us to obtain light enhancements by the SP coupling for the first time. By this configuration, we can avoid an absorption loss at the metal layer and obtain an effective light extraction from SPP at the interface.

Fig. 6(b) shows typical PL spectra from an InGaN/GaN QWs separated from $\mathrm{Ag}, \mathrm{Al}$, and $\mathrm{Au}$ layers by $10 \mathrm{~nm} \mathrm{GaN}$ spacers. Huge enhancements of PL spectra of both blue and green emissions were observed from the Ag- or Al-coated samples. The PL peak of the uncoated wafer is normalized to 1. A 14-fold enhancement of blue emission is observed from the Ag-coated sample, whereas 8-fold enhancements are obtained from Alcoated sample. These enhancements were a little reduced for the sample with green emission. We believe that these PL enhancements should be attributed to the energy transfer between QWs and SPs. No such enhancements were obtained from samples coated with $\mathrm{Au}$, as its well-known plasmon resonance occurs only at longer wavelengths.

We have several evidences to support the contribution of the SPs to obtained PL enhancements. Fig. 7(a) shows the enhancement ratios of PL intensities with metal layers separated from the QWs by $10 \mathrm{~nm}$ spacers as a function of wavelength. We find that the enhancement ratio increases at shorter wavelengths for Ag samples, whereas it is independent of wavelength for Al-coated samples. The PL enhancement after coating with Ag
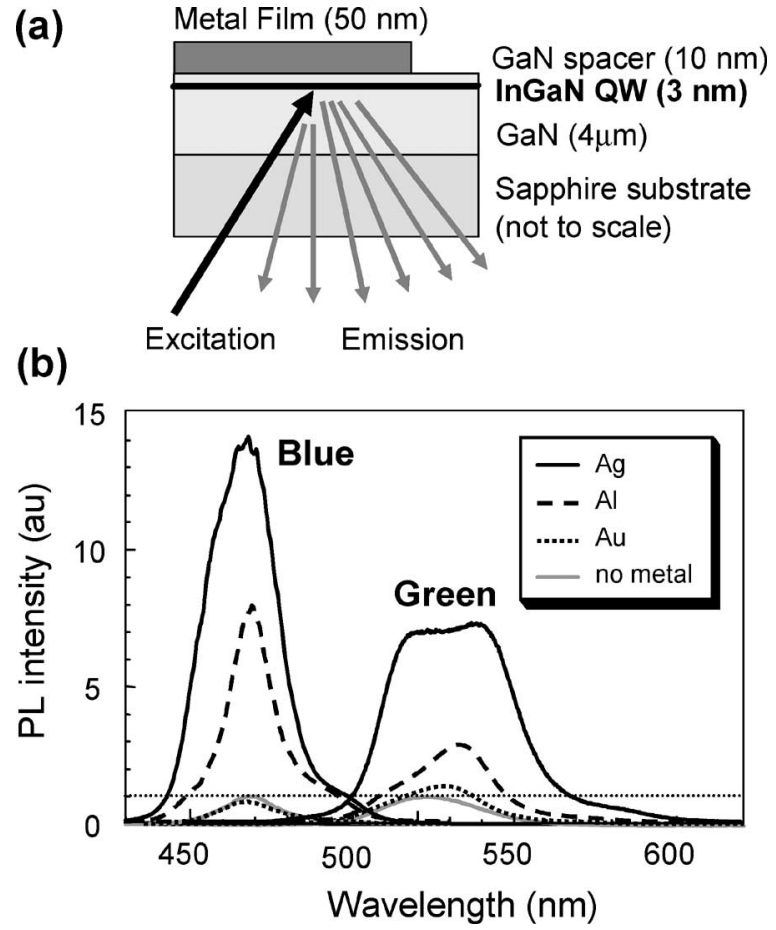

Fig. 6. (a) Sample structure of InGaN/GaN QW and excitation/emission configuration of PL measurement. (b) SP-enhanced blue and green emissions from InGaN/GaN QWs coated with Ag, Al, or Au. The PL peak intensity of uncoated InGaN/GaN QW was normalized to 1 .

and $\mathrm{Al}$ can be attributed to strong interaction with SPs. Fig. 7(b) shows the dispersion diagrams of SPP modes on metal/GaN surfaces calculated by (1). The SP frequency $\left(\omega_{\mathrm{SP}}\right)$ at $\mathrm{GaN} / \mathrm{Ag}$ is $2.84 \mathrm{eV}(437 \mathrm{~nm})$. Thus, $\mathrm{Ag}$ is suitable for SP coupling to blue emission, and we attribute the large increases in the PL intensity from Ag-coated samples to such resonant SP excitation. In contrast, $\omega_{\mathrm{SP}}$ at $\mathrm{GaN} / \mathrm{Au}$ is $2.462 \mathrm{eV}(537 \mathrm{~nm})$, and no measurable enhancement is observed in Au-coated InGaN emitters as the SP and QW energies do not match. In the case of $\mathrm{Al}, \omega_{\mathrm{SP}}$ is $5.50 \mathrm{eV}(225 \mathrm{~nm})$, and the real part of the dielectric constant is negative over a wide wavelength region for visible light. Thus, a substantial and useful PL enhancement is observed in Al-coated samples, although the energy match is not ideal at $470 \mathrm{~nm}$ and a better overlap is expected at shorter wavelengths. The clear correlation between Fig. 7(a) and (b) suggests that the obtained emission enhancement with $\mathrm{Ag}$ and $\mathrm{Al}$ are due to the SP coupling. The SP enhancement effects of the green emissions were not so large as those of the blue emissions [see Fig. 6(b)] because the green spectral region is far from the SP frequency.

PL intensities of Al- and Ag-coated samples were also found to depend strongly on the distance between QWs and the metal layers, in contrast to Au-coated samples. Fig. 8 compares integrated PL enhancement ratios for three different GaN spacer thicknesses (of 10, 40, and $150 \mathrm{~nm}$ ) for $\mathrm{Ag}, \mathrm{Al}$, and $\mathrm{Au}$ coatings for (a) blue and (b) green emissions. $\mathrm{Al}$ and $\mathrm{Ag}$ samples show exponential decreases in the PL intensity as the spacer thickness is increased, whereas no such reduction was measured in Au-coated QWs. This spacer layer dependence of the PL 
(a)

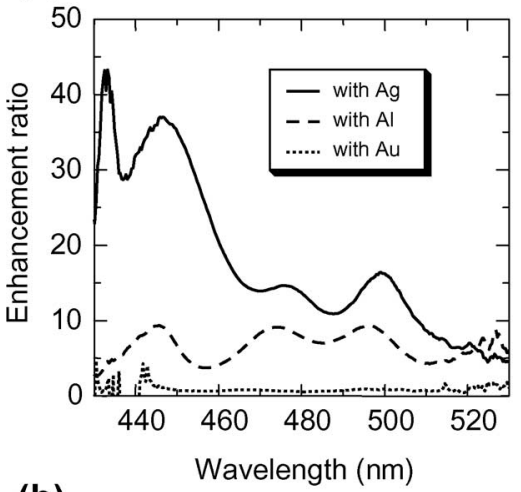

(b)

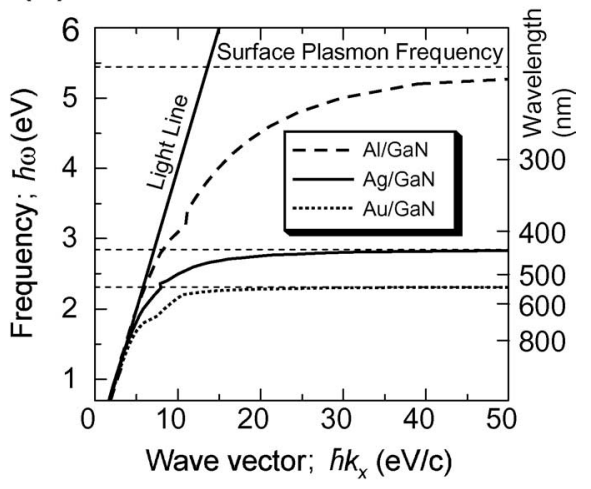

Fig. 7. (a) PL enhancement ratios at several wavelengths for the same samples as Fig. 6(b). (b) Dispersion diagrams of the SPP at Al/GaN, Ag/GaN, and $\mathrm{Au} / \mathrm{GaN}$ interfaces.

enhancement ratios matches our models of SP-QW coupling, as the SPP should be localized at the metal/dielectric interface and exponentially decays with distance from the metal surface. Only electron-hole pairs located within the near field of the surface can couple to the SPP mode, and this penetration depth $\left(d_{z}\right)$ of the SP fringing field into the semiconductor is given by

$$
d_{z}(\omega)=\frac{c}{\omega} \sqrt{\frac{\varepsilon_{2}(\omega)+\varepsilon_{1}^{\prime}(\omega)}{-\varepsilon_{2}(\omega)^{2}}} .
$$

Fig. 8 shows a good agreement between these calculated penetration depths (lines) and measured values of the PL enhancement (symbols) for Ag- and Al-coated samples.

\section{SP Coupling Mechanism}

Possible mechanism of the QW-SP coupling and the light extraction is shown in Fig. 9. First, electron-hole pairs are generated in the QW by photopumping or electrical pumping. For uncoated samples, these carriers are terminated by the radiative or nonradiative recombination rates, and the IQE is determined by the ratio of these two values [see Fig. 9(a)]. When a metal layer is grown within the near field of the active layer, and when the bandgap energy of InGaN active layer is close to the energy of the SP frequency at the metal/semiconductor interface, the QW energy can transfer to the SP. Then, electron-hole recombination may produce SPPs instead of photons or phonons. Since the density of states of SPP mode is much larger, the QW-SP (a)

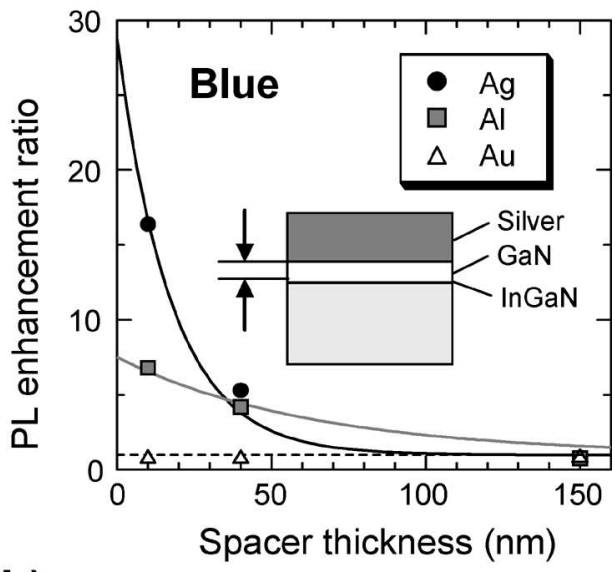

(b)

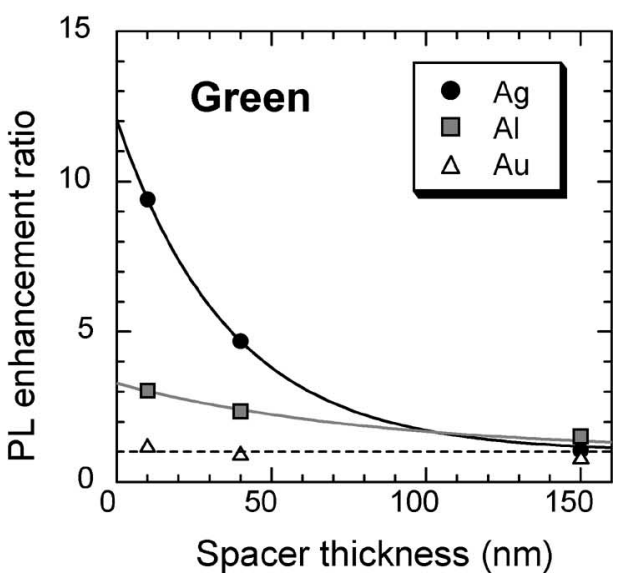

Fig. 8. Integrated $\mathrm{PL}$ enhancement ratios for samples with $\mathrm{Ag}, \mathrm{Al}$, and $\mathrm{Au}$ are plotted against the thicknesses of GaN spacers for (a) blue and (b) green emissions. The solid lines are the calculated values of the penetration depths using (2).

coupling rate $\left(k_{\mathrm{SPC}}\right)$ should be very fast, and this new path of a recombination can increase the spontaneous emission rate and the IQE.

The SP coupling in LED devices may be considered detrimental to the emission efficiency, because the SP is a nonradiative wave. If the metal/semiconductor surface were perfectly flat, it would be difficult to extract light from the SPP mode, and the SP energy would be thermally dissipated. However, the SPP energy can be extracted as light by providing roughness or nanostructuring the metal layer. Such roughness allows SPPs of high momentum to scatter, lose momentum, and couple to radiated light [34]. The few tens of nanometer-sized roughness in the Ag surface layer can be obtained by controlling the evaporation conditions or by nanofabrication to obtain the high photon extraction efficiencies. Such roughness in the metal layer was observed from higher magnification SEM images of the Ag-coated surface [see Fig. 10(a)] and the original GaN surface [see Fig. 10(b)]. The length scale of the roughness of the $\mathrm{Ag}$ surface was determined to be a few hundred nanometers. Fig. 10(c) shows the microscopic PL image that includes original and Ag-coated regions. Obviously, the emission intensity 


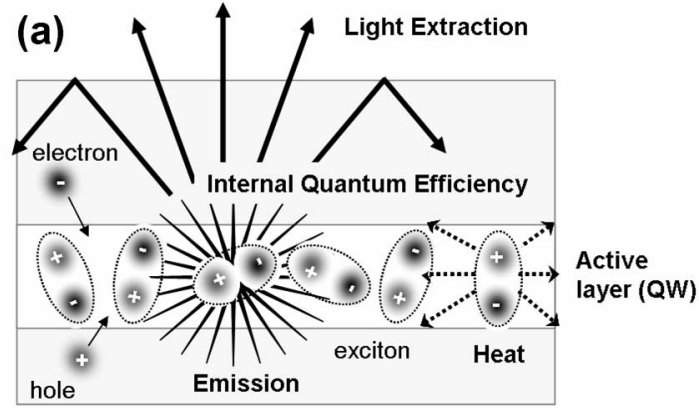

(b)

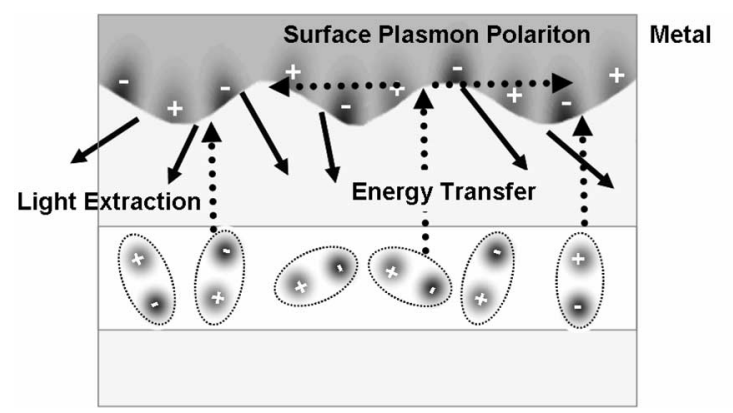

Fig. 9. Schematic diagram of (a) electron-hole recombination and (b) QW-SP coupling mechanism.

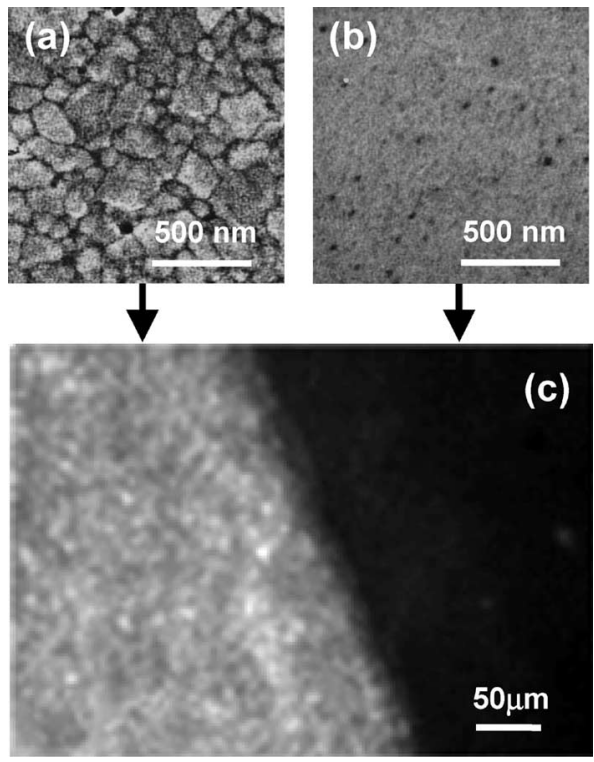

Fig. 10. (a) SEM image of the 50-nm Ag film evaporated on GaN. (b) SEM image of the uncoated GaN surface. (c) Microscopic PL image including the areas of (a) and (b). All samples used were coated onto InGaN/GaN QWs with $10 \mathrm{~nm}$ GaN spacers.

from the Ag-coated region is much brighter and has inhomogeneous patterns in the image. This pattern structure in the PL image should be due to the roughness of the Ag surface because the SPP was extracted as light at the Ag structures.

In order to evaluate the SP coupling mechanism we proposed, we employed a 3-D finite-difference time-domain (3-D FDTD) method to reproduce the coupling processes between electron-hole pairs, SPPs, and photons. To perform 3-D FDTD (a)

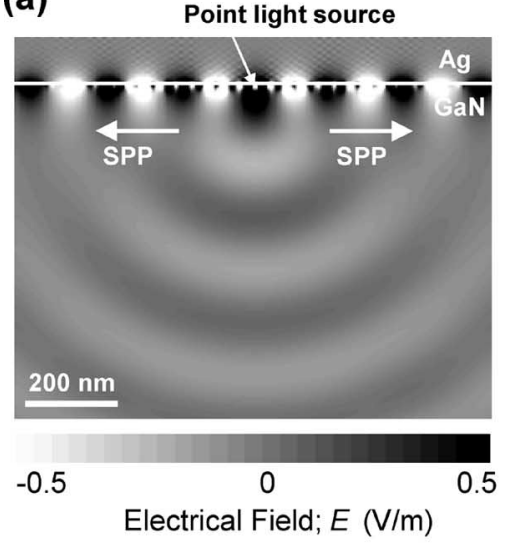

(b)

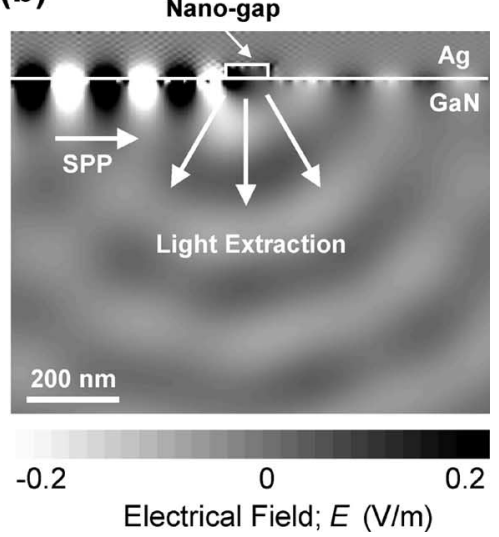

Fig. 11. 3-D FDTD simulations of generation and light extraction of SPPs. (a) SSP was generated and propagated on the interface when a point light source was located on the interface. (b) Generated SSP was extracted as light at the gap in the metal layer.

simulations, we used "Poynting for optics" (Fujitsu Company), which is known to be very suitable to simulate SPP modes [35]. A polarized plane wave with $525 \mathrm{~nm}$ wavelength and $1 \mathrm{~V} / \mathrm{m}$ amplitude was used as a point light source, which is the assumption of an electron-hole pair. Fig. 11 shows the calculated spatial distribution of the electromagnet field around the $\mathrm{Ag} / \mathrm{GaN}$ interface. If the point light source was located near the interface, the SPP was generated and propagated within the interface [see Fig. 11(a)] while it was not excited if the point light source was far from the interface. This result suggests that the SPP mode can be generated easily by direct energy transfer from electronhole pairs without any special structures. The generated SPP mode can be coupled to photon if there is a nanosized gap structure at the interface [see Fig. 11(b)]. Then, generated SPP can be extracted from the interface as light, and as a consequence, the emission efficiency is increased. These calculations support our proposed SP coupling model.

\section{Improvements of Internal Quantum Efficiencies}

Our proposed model suggests that the SP coupling should increase IQEs of emissions. In order to obtain the IQE values to separate the SP enhancement from other possible effects, we have measured the temperature dependence of the PL intensity. Fig. 12 shows Arrhenius plots of the integrated PL intensities 


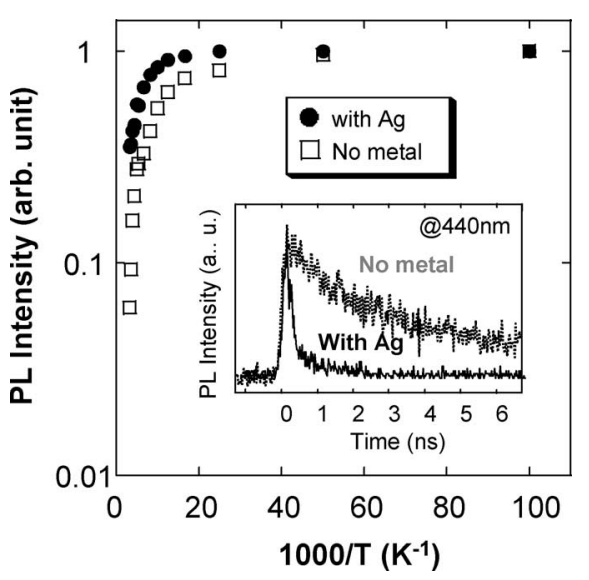

Fig. 12. Arrhenius plots of the integrated PL intensities of InGaN/GaN QWs with $\mathrm{Ag}$ and uncoated sample with $10 \mathrm{~nm} \mathrm{GaN}$ spacers. PL integrated intensities at $10 \mathrm{~K}$ were normalized to 1 . (Inset) PL decay profiles of uncoated and $\mathrm{Ag}$ coated $\mathrm{InGaN} / \mathrm{GaN} \mathrm{QW}$ at $440 \mathrm{~nm}$.

from InGaN QWs separated from Ag films by $10 \mathrm{~nm}$ spacers, and compares these to uncoated samples. The IQE values from uncoated QWs were estimated as $6 \%$ at room temperature by assuming $\eta_{\text {int }} \sim 100 \%$ at $10 \mathrm{~K}$. These IQE values increased to $40 \%$ after Ag coating, which is explainable by spontaneous recombination rate enhancements through SP coupling. This suggests that the spontaneous emission rate becomes much faster by the SP coupling. We investigated the direct observation of SP coupled spontaneous emission rate by using the TRPL measurements [36]. Inset of Fig. 12 shows the PL decay profiles of uncoated and Ag-coated InGaN/GaN QW samples emitters at $440 \mathrm{~nm}$. The emission lifetime of Ag-coated sample ( $200 \mathrm{ps})$ was 30 times faster than that of uncoated sample ( $\sim 6 \mathrm{~ns})$.

The original spontaneous emission rate $\left(k_{\mathrm{PL}}\right)$ is attributed to the radiative $\left(k_{\mathrm{rad}}\right)$ and nonradiative $\left(k_{\mathrm{non}}\right)$ recombination rates of the electron-hole pairs as $k_{\mathrm{PL}}=k_{\mathrm{rad}}+k_{\mathrm{non}}$. By the SP coupling, the spontaneous emission rate should be increased to $k_{\mathrm{PL}}^{*}$ by the SP coupling rate $\left(k_{\mathrm{SPC}}\right)$ as $k_{\mathrm{PL}}^{*}=k_{\mathrm{rad}}+k_{\text {non }}+$ $k_{\mathrm{SPC}}$. All time profiles could be fitted to single exponential functions, and the obtained $k_{\mathrm{PL}}$ and $k_{\mathrm{PL}}^{*}$ values were plotted against wavelength in Fig. 13(a). The emission rates of Agcoated sample were much faster than those of the uncoated sample and strongly depend on the wavelength. This difference becomes dramatically larger at the shorter wavelength region. The spontaneous emission rates in the SPP mode $\left(k_{\mathrm{SPC}}\right)$ depend on the density of states of the SPP by Fermi's golden rule [31], [32]. The density of states of the SPP mode is proportional to $d k / d \omega$, which can be obtained by the dispersion curve [see Fig. 7(b)]. In Fig. 13(a), $d k / d \omega$ is also plotted as the solid line. The SP coupling rate should be almost equal to the PL decay rate of Ag-coated sample because these values were much larger than the values of the PL decay rate without Ag. Fig. 13(a) shows that the wavelength dependence of the SP coupling rates is very similar to that of $d k / d \omega$.

Fig. 13(b) shows the enhanced IQEs $\left(\eta_{\text {int }}^{*}\right)$ estimated by the ratios between $k_{\mathrm{PL}}$ and $k_{\mathrm{PL}}^{*}$ under the assumption that the light extraction from the generated SPP mode is almost $100 \%$. The SP coupling becomes remarkable when the energy is near to the
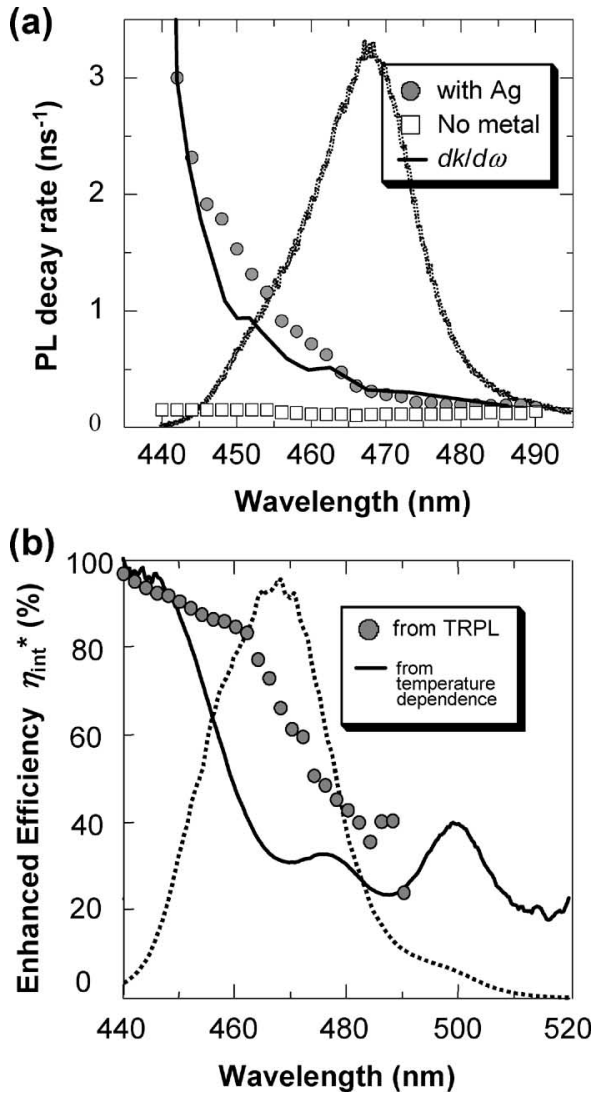

Fig. 13. (a) Spontaneous emission rates of InGaN/GaN with/without Ag layer plotted against wavelength. The solid line is $d k / d \omega$ of the SPP mode at the $\mathrm{Ag} / \mathrm{GaN}$ interface obtained by the dispersion curve [see Fig. 7(b)]. The dashed line is the emission spectrum. (b) Wavelength-dependent internal quantum efficiencies of the InGaN/GaN with $\mathrm{Ag}$ layer with $10 \mathrm{~nm} \mathrm{GaN}$ spacers estimated by the ratios of the emission rates (marks) and the temperature dependence of the PL intensities (line). The dotted black line is the PL spectrum of the same sample.

SP frequency described in Fig. 7(b) as $2.84 \mathrm{eV}(437 \mathrm{~nm})$. At this shorter wavelength region, the SP coupling rates are much faster than the radiative or nonradiative recombination rates of electron-hole pairs $\left(k_{\mathrm{SPC}} \gg k_{\mathrm{rad}}+k_{\mathrm{non}}\right)$, and the values of $\eta_{\text {int }}^{*}$ can reach to almost $100 \%$. Wavelength-dependent $\eta_{\text {int }}^{*}$ values were also estimated from the temperature dependence of the PL intensities (see Fig. 12) and plotted in Fig. 13(b) (solid line). Both data show similar behavior, and the discrepancy of each datum should be due to the light extraction probability from the SPP. $\eta_{\text {int }}^{*}$ estimated by the temperature-dependent measurements of the PL intensities should include the damping energy loss of the SPP. An important fact is that the both $\eta_{\text {int }}^{*}$ values can reach to almost $100 \%$ at the shorter wavelength region. This suggests that the SP coupling method can provide $100 \%$ efficiencies of emission if the energy matching condition between QW and SP frequency is well satisfied.

\section{Potentials of Plasmon Photonics}

\section{A. Superbright Nanophotonic and Plasmonic LEDs}

We described powerful methods to improve emission efficiencies of InGaN/GaN QWs based on nanophotonics and plasmonics. Both methods increase IQEs by increasing spontaneous 


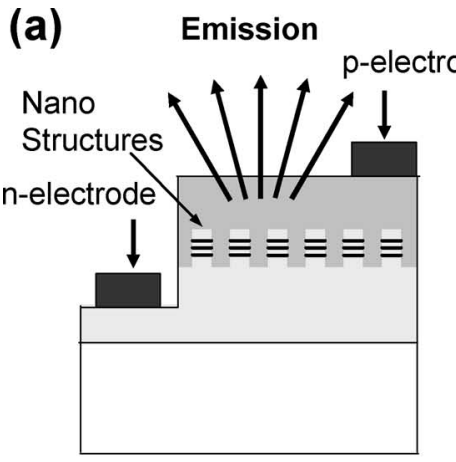

(b)

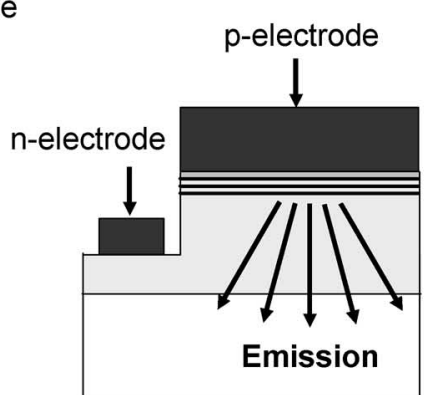

(c) Emission

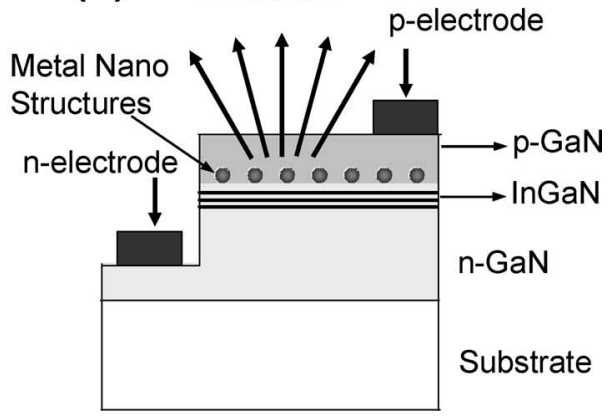

Fig. 14. Possible device structures of high-efficiency LEDs based on nanophotonics and plasmonics with electrical pumping. (a) p-GaN layer was grown on nanostructured InGaN QWs (b) Metal electrode is located a few nanometers above the active layer. (c) Metal particles are embedded a few nanometers above the active layer.

emission rates. This suggests that these methods should be applicable for electrical pumping because the IQEs do not depend on the pumping method. So, now we try to make superbright nanophotonic and plasmonic LEDs by electrical pumping. Possible device structures of high-efficiency LEDs are shown in Fig. 14.

Fig. 14(a) shows the nanostructure-enhanced InGaN-LED. p-GaN layer is grown onto the nanofabricated InGaN active layer, such as nanodisks or nanopillars. Actual devices based on this structure are now under developments. Fig. 14(b) shows the simple structure using a usual LED structure with a p-n junction. The metal layer can be used both as an electrical contact and for exciting plasmons. The important point of this structure is that the distance between the metal surface and the InGaN QW must be very close to get a good SP coupling. The p-type GaN layer must be very thin around $10-20 \mathrm{~nm}$, because the PL enhancement ratios exponentially decay with increasing of the thickness of the GaN spacer layer (see Fig. 8). This feature makes the device application of the SP coupling so difficult. We already fabricated the device structure shown in Fig. 14(b), but we were not able to obtain a huge enhancement of emission. There are two reasons: first, p-doping was very difficult into 10-nm-thick GaN layer. Second, we could not get a good ohmic contact because the p-GaN layer is too thin. Another possible structure of a plasmonic LED is shown in Fig. 14(c). In this structure, the metal layer for electrode and for SP coupling is different. The SP coupling should happen at the metal particles embedded just above a QW layer in an LED wafer. Fabrication of this structure is now in progress.

Quite recently, a few groups reported about the SP-enhanced LEDs based on our technique. Yeh et al. reported the SP coupling effect in an InGaN/GaN single-QW LED structure [37]. Their LED structure has a 10-nm p-type AlGaN current blocking layer and a 70-nm p-type GaN layer between the metal surface and the $\mathrm{InGaN}$ QW layer. The total distance is $80 \mathrm{~nm}$, which is too far to obtain an effective SP coupling. Because of this reason, they obtained only 1.5 -fold enhancement of the emission. Kwon et al. also reported a plasmonic LED, which has structure similar to the one shown in Fig. 14(c) [38]. They put $\mathrm{Ag}$ particles on the InGaN QW layer first, and overgrew a $\mathrm{GaN}$ layer above the Ag particles. However, a large amount of $\mathrm{Ag}$ particles was lost by high temperature of the crystal growth, and only 3\% particles remained. Therefore, they obtained only 1.3fold enhancement of the emission. These small enhancement ratios are not good enough for device application. Therefore, a high-efficiency LED structure based on plasmonics is not yet achieved.

\section{B. Further Advantages of the Plasmonic LEDs}

Our proposed plasmonic LEDs should have several very important advantages. We found that the SP coupling enhances the IQEs, and it reached almost $100 \%$ at the best matched wavelength. If we can control the SP frequency and obtain the best matching condition between the emission wavelength and the SP frequency, we can increase both $\eta_{\text {int }}^{*}$ and $C_{\text {ext }}$ to $100 \%$ at any wavelength. It is the perfect efficiency, and will lead to full color devices and natural white LEDs by using only InGaN/GaN material. Tuning of SP coupling should be available by choosing the appropriate metal, metal mixture alloys, multiple layers, or nanostructures. For example, we could further improve the green emission of InGaN by fabricating the nanograting structures of gold layer by electron beam lithography and Ar ion milling [39]. Theoretical study was also reported by Paiella to tune the SP frequency by using metallodielectric multiple layers [40].

Another important advantage is that the SP-enhanced technique can be applied not only to InGaN-based materials but also to various materials that suffer from low quantum efficiencies. Indeed, we obtained similar obvious enhancements of both PL intensities and emission rates for several materials, such as organic dye molecules doped in polymers [41], light-emitting polymer with $\pi$-electron conjugation [42], and CdSe-based quantum dot nanocrystals [43]. Moreover, we obtained a large PL enhancement for silicon nanocrystals in silicon dioxide media. [44] Usually, the emission efficiencies of such indirect semiconductors are quite low, but by using the SP coupling, it is possible to increase these efficiencies up to values as large as those of direct compound semiconductors. We believe that the SP coupling technique would provide extremely bright silicon-based light-emitting devices, which could be very cheap to make and easy to process, and would become widely used light source instead of fluorescent light bulbs in the near future. Ultimately, this technique could enable new bright light emitters based on light emission through inelastic tunneling 
within metal/insulator/metal (MIM) structures without relying on semiconductor emitting materials.

Similar plasmonic design should also be applicable to devices based on nonlinear optical materials, photo detectors, solar cells, waveguides, optical modulators, and other optical and electric devices. Moreover, several future possibilities of plasmonics have been proposed recently, e.g., the plasmonic metamaterials [45] with negative refractive index at visible regions, the plasmonic therapy for cancer, and the optical cloaking technology [46] based on plasmonics. Undoubtedly, plasmonics is becoming a key technology in wider fields and will attract much more attentions in near future.

\section{CONCLUSION}

We described that the emission efficiencies of $\mathrm{InGaN} / \mathrm{GaN}$ QWs can be dramatically enhanced by fabricating nanostructures or using energy coupling to the SPP generated at metal surfaces. These are very promising methods for developing high-efficiency LEDs. Especially, the SP coupling method is very simple and easy, and has many advantages. Even when using unpatterned metal layers, the SP energy can be extracted by the submicrometer-scale roughness on the metal surface. High-efficiency light emission is predicted for optically as well as electrically pumped light emitters, because the mechanism is not related to the pumping method. If the SP frequency is carefully selected to match the QW emission frequency, the increase of the density of states can result in large enhancements of the IQEs and the spontaneous emission rate. The SP-enhanced technique can be applied not only to III-V materials, but also to other various materials that suffer from low quantum efficiencies, which include the indirect semiconductors. We believe that this SP coupling technique will result in the realization of superbright plasmonic LEDs, and presumably become the dominant white light sources alternative to conventional incandescent and fluorescent light bulbs.

\section{ACKNOWLEDGMENT}

The authors wish to thank Prof. A. Scherer of California Institute of Technology for valuable discussions and support. They also thank Mr. A. Shavartser, Dr. T. D. Neal, Dr. S. Vyawahare, and G. Maltezos (California Institute of Technology), Dr. A. Kaneta and Dr. K. Nishizuka (Kyoto University), Mr. I. Niki, Dr. Y. Narukawa, and Dr. T. Mukai (Nichia Corporation), and Prof. R. Micheletto (Yokohama City University) for their help.

\section{REFERENCES}

[1] S. Nakamura, T. Mukai, M. Senoh, S. Nagahama, and N. Iwasa, "P-GaN/N-InGaN/N-GaN double-heterostructure blue-light-emitting diodes," Jpn. J. Appl. Phys., vol. 32, no. 1A/B, pp. L8-L11, Jan. 1993.

[2] K. Bando, K. Sakano, Y. Noguchi, and Y. Shimizu, "Development of highbright and pure-white LED lamps," J. Light Vis. Environ., vol. 22, no. 1, pp. 2-5, 1998

[3] Y. Narukawa, J. Narita, T. Sakamoto, K. Deguchi, T. Yamada, and T. Mukai, "Improvement of luminous efficiency in white light emitting diodes by reducing a forward-bias voltage," Jpn. J. Appl. Phys., vol. 46, no. 36-40, pp. L963-L965, Oct. 2007.
[4] M. Yamada, T. Mitani, Y. Narukawa, S. Shioji, I. Niki, S. Sonobe, K. Deguchi, M. Sano, and T. Mukai, "InGaN-based near-ultraviolet and blue-light-emitting diodes with high external quantum efficiency using a patterned sapphire substrate and a mesh electrode," Jpn. J. Appl. Phys., vol. 41, no. 12A, pp. L1431-L1433, Dec. 2002.

[5] J. J. Wierer, M. R. Krames, J. E. Epler, N. F. Gardner, M. G. Craford, J. R. Wendt, J. A. Simmons, and M. M. Sigalas, "InGaN/GaN quantumwell heterostructure light-emitting diodes employing photonic crystal structures," Appl. Phys. Lett., vol. 84, no. 19, pp. 3885-3886, May 2004.

[6] T. Mukai, K. Takekawa, and S. Nakamura, "InGaN-based blue lightemitting diodes grown on epitaxially laterally overgrown GaN substrates," Jpn. J. Appl. Phys., vol. 37, no. 7B, pp. L839-L841, Jul. 1998.

[7] T. Mukai and S. Nakamura, "Ultraviolet InGaN and GaN single-quantumwell-structure light-emitting diodes grown on epitaxially laterally overgrown GaN substrates," Jpn. J. Appl. Phys., vol. 38, no. 10, pp. 57355739, Oct. 1999.

[8] S. Chichibu, T. Azuhata, T. Sota, and S. Nakamura, "Spontaneous emission of localized excitons in InGaN single and multiquantum well structures," Appl. Phys. Lett., vol. 69, no. 27, pp. 4188-4190, Dec. 1996.

[9] Y. Narukawa, Y. Kawakami, M. Funato, S. Fujita, S. Fujita, and S. Nakamura, "Role of self-formed InGaN quantum dots for exciton localization in the purple laser diode emitting at $420 \mathrm{~nm}$," Appl. Phys. Lett., vol. 70, no. 8, pp. 981-983, Feb. 1997.

[10] T. Takeuchi, S. Sota, M. Katsuragawa, M. Komori, H. Takeuchi, H. Amano, and I. Akasaki, "Quantum-confined Stark effect due to piezoelectric fields in GaInN strained quantum wells," Jpn. J. Appl. Phys., vol. 36, no. 4A, pp. L382-L385, Apr. 1997.

[11] P. Walterelt, O. Brandt, A. Trampert, H. T. Grahn, J. Menniger, M. Ramsteiner, M. Reiche, and K. H. Ploog, "Nitride semiconductors free of electrostatic fields for efficient white light-emitting diodes," Nature, vol. 406, pp. 865-868, Aug. 2000.

[12] K. Nishizuka, M. Funato, Y. Kawakami, S. Fujita, Y. Narukawa, and T. Mukai, "Efficient radiative recombination from $\langle 112-2\rangle$ oriented $\mathrm{In}_{x} \mathrm{Ga}_{1-x} \mathrm{~N}$ multiple quantum wells fabricated by the regrowth technique," Appl. Phys. Lett., vol. 85, no. 15, pp. 3122-3124, Oct. 2004.

[13] T. Sugahara, M. Hao, T. Wang, D. Nakagawa, Y. Naoi, K. Nishino, and S. Sakai, "Role of dislocation in InGaN phase separation," Jpn. J. Appl. Phys., vol. 37, no. 10B, pp. L1195-L1198, Oct. 1998.

[14] S. Chichibu, K. Wada, and S. Nakamura, "Spatially resolved cathodoluminescence spectra of InGaN quantum wells," Appl. Phys. Lett., vol. 71, no. 16, pp. 2346-2348, Oct. 1997.

[15] K. Okamoto, J. Choi, Y. Kawakami, M. Terazima, T. Mukai, and S. Fujita, "Submicron-scale photoluminescence of InGaN/GaN probed by confocal scanning laser microscopy," Jpn. J. Appl. Phys., vol. 43, no. 2, pp. 839840, Feb. 2004

[16] K. Okamoto, A. Kaneta, Y. Kawakami, S. Fujita, J. Choi, M. Terazima, and T. Mukai, "Confocal micro-photoluminescence of InGaN-based light emitting diodes," J. Appl. Phys., vol. 98, pp. 064503-1-064503-7, Sep. 2005.

[17] A. Kaneta, K. Okamoto, Y. Kawakami, S. Fujita, G. Marutsuki, Y. Narukawa, and T. Mukai, "Spatial and temporal luminescence dynamics in an $\operatorname{In}_{x} \mathrm{Ga}_{1-x} \mathrm{~N}$ single quantum well probed by near-field optical microscopy," Appl. Phys. Lett., vol. 81, no. 23, pp. 4353-4355, Dec. 2002.

[18] K. Okamoto, A. Scherer, and Y. Kawakami, "Near-field scanning optical microscopic transient lens for carrier dynamics study in InGaN/GaN," Appl. Phys. Lett., vol. 87, pp. 161104-1-161104-3, Oct. 2005.

[19] A. Kaneta, M. Funato, and Y. Kawakami, "Nanoscopic recombination processes in InGaN/GaN quantum wells emitting violet, blue, and green spectra," Phys. Rev. B, vol. 78, pp. 125317-1-125317-6, Sep. 2008.

[20] A. Scherer, J. L. Jewell, Y. H. Lee, J. P. Harbison, and L. T. Florez, "Fabrication of microlasers and microresonator optical switches," Appl. Phys. Lett., vol. 55, no. 26, pp. 2724-2726, Dec. 1989.

[21] Y. Kawakami, A. Kaneta, L. Su, Y. Zhu, K. Okamoto, M. Funato, A. Kikuchi, and K. Kishino, "Radiative and nonradiative recombination processes in InGaN GaN nano-pillars fabricated by post-growth chemically assisted ion beam etching," submitted for publication.

[22] M. Nirmal, B. O. Dabbousi, M. G. Bawendi, J. J. Macklin, J. K. Trautman, T. D. Harris, and L. E. Brus, "Fluorescence intermittency in single cadmium selenide nanocrystals," Nature, vol. 383, pp. 802-804, Oct. 1996.

[23] R. Micheletto, M. Abiko, A. Kaneta, Y. Kawakami, Y. Narukawa, and T. Mukai, "Observation of optical instabilities in the photoluminescence of InGaN single quantum well," Appl. Phys. Lett., vol. 88, pp. 0611181-061118-3, Feb. 2006. 
[24] M. Yoshizawa, A. Kikuchi, M. Mori, N. Fujita, and K. Kishino, "Growth of self-organized $\mathrm{GaN}$ nanostructures on $\mathrm{Al}_{2} \mathrm{O}_{3}(0001)$ by RF-radical source molecular beam epitaxy," Jpn. J. Appl. Phys., vol. 36, no. 4B, pp. L459L462, Apr. 1997.

[25] H. Raether, Surface Plasmons on Smooth and Rough Surfaces and on Gratings. New York: Springer-Verlag, 1988.

[26] S. A. Maier, Plasmonics: Fundamentals and Applications. New York: Springer-Verlag, 2007.

[27] W. L. Barnes, A. Dereux, and T. W. Ebbesen, "Surface plasmon subwavelength optics," Nature, vol. 424, pp. 824-830, Aug. 2003.

[28] J. Dostalek, J. Homola, S. Jiang, and J. Ladd, Surface Plasmon Resonance Based Sensors. New York: Springer-Verlag, 2006.

[29] A. Köck, E. Gornik, M. Hauser, and M. Beinstingl, "Strongly directional emission from AlGaAs/GaAs light-emitting diode," Appl. Phys. Lett., vol. 57, no. 22, pp. 2327-2329, Nov. 1990.

[30] J. Vuckovic, M. Loncar, and A. Scherer, "Surface plasmon enhanced lightemitting diode," IEEE J. Quantum Electron., vol. 36, no. 10, pp. 11311144, Oct. 2000.

[31] I. Gontijo, M. Borodisky, E. Yablonvitch, S. Keller, U. K. Mishra, and S. P. DenBaars, "Enhancement of spontaneous recombination rate in a quantum well by resonant surface plasmon coupling," Phys. Rev. B, vol. 60, no. 16, pp. 11564-11567, Oct. 1999.

[32] A. Neogi, C.-W. Lee, H. O. Everitt, T. Kuroda, A. Tackeuchi, and E. Yablonvitch, "Enhancement of spontaneous recombination rate in a quantum well by resonant surface plasmon coupling," Phys. Rev. B, vol. 66, pp. 153305-1-153305-4, Oct. 2002.

[33] K. Okamoto, I. Niki, A. Shvartser, Y. Narukawa, T. Mukai, and A. Scherer, "Surface-plasmon-enhanced light emitters based on InGaN quantum wells," Nature Mater, vol. 3, pp. 601-605, Sep. 2004.

[34] W. L. Barnes, "Turning the tables on surface plasmons," Nature Mater., vol. 3, pp. 588-589, Sep. 2004.

[35] W. Nomura, M. Ohtsu, and T. Yatsui, "Nanodot coupler with a surface plasmon polariton condenser for optical far/near-field conversion," Appl. Phys. Lett., vol. 86, pp. 181108-1-181108-3, May 2005.

[36] K. Okamoto, I. Niki, A. Scherer, Y. Narukawa, T. Mukai, and Y. Kawakami, "Surface plasmon enhanced spontaneous emission rate of InGaN/GaN quantum wells probed by time-resolved photoluminescence spectroscopy," Appl. Phys. Lett., vol. 87, pp. 071102-1-071102-3, Aug. 2005.

[37] D.-M. Yeh, C.-F. Huang, C.-Y. Chen, Y.-C. Lu, and C. C. Yanga, "Surface plasmon coupling effect in an InGaN/GaN single-quantum-well lightemitting diode," Appl. Phys. Lett., vol. 91, pp. 171103-1-171103-3, Oct. 2007.

[38] M.-K. Kwon, J.-Y. Kim, B.-H. Kim, I.-K. Park, C.-Y. Cho, C. C. Byeon, and S.-J. Park, "Surface-plasmon-enhanced light-emitting diodes," Adv. Mater, vol. 20, no. 7, pp. 1253-1257, Apr. 2008

[39] K. Okamoto, I. Niki, A. Shvartser, G. Maltezos, Y. Narukawa, T. Mukai, Y. Kawakami, and A. Scherer, "Surface plasmon enhanced bright light emission from InGaN/GaN," Phys. Stat. Sol. (a), vol. 204, no. 6, pp. $2103-$ 2107, Jun. 2007.

[40] R. Paiella, "Tunable surface plasmons in coupled metallo-dielectric multiple layers for light-emission efficiency enhancement," Appl. Phys. Lett., vol. 87, pp. 111104-1-111104-3, Sep. 2005.

[41] T. D. Neal, K. Okamoto, and A. Scherer, "Surface plasmon enhanced emission from dye doped polymer layers," Opt. Exp., vol. 13, no. 14, pp. 5522-5527, Jul. 2005.

[42] T. D. Neal, K. Okamoto, A. Scherer, M. S. Liu, and A. K.-Y. Jen, "Timeresolved photoluminescence spectroscopy of surface-plasmon-enhanced light emission from conjugate polymers," Appl. Phys. Lett., vol. 89, pp. 221106-1-221106-3, Nov. 2006.
[43] K. Okamoto, S. Vyawahare, and A. Scherer, "Surface plasmon enhanced bright emission from CdSe quantum dots nanocrystal," J. Opt. Soc. Amer. $B$, vol. 23, no. 8, pp. 1674-1678, Aug. 2006.

[44] K. Okamoto, A. Scherer, and Y. Kawakami, "Surface plasmon enhanced light emission from semiconductor materials," Phys. Stat. Sol. (c), vol. 5, no. 9, pp. 2822-2824, Jun. 2008.

[45] N. Fang, H. Lee, C. Sun, and X. Zhang, "Sub-diffraction-limited optical imaging with a silver superlens," Science, vol. 308, pp. 534-537, Apr. 2005.

[46] H. A. Atwater, "The promise of plasmonics," Sci. Amer, vol. 296, pp. 5663, Apr. 2007

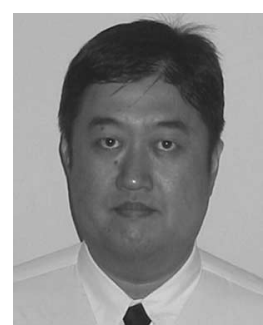

Koichi Okamoto received the B.S. degree from the Department of Chemical Engineering, Doshisha University, Kyoto, Japan, in 1991, and the M.S. and Ph.D. degrees from the Department of Chemistry, Kyoto University, Kyoto, in 1994 and 1998, respectively.

From 1998 to 2000, he was a Researcher in the Venture Business Laboratory, Kyoto University, where he has been a Research Associate Professor of Electronic Science and Engineering since September 2009. From 1998 to 2000, he was a Research Fellow of the Japan Society for the Promotion of Science (JSPS). From 2001 to 2005, he was a Postdoctoral Scholar of Electrical Engineering at California Institute of Technology, where he was a Senior Research Fellow of Physics from 2005 to 2007. Since 2006, he has also been a Precursory Research for Embryonic Science and Technology (PRESTO) Researcher at Japan Science and Technology Agency (JST), Kawaguchi, Japan. His current research interests include developing new optical materials and devices based on nanophotonics and plasmonics.

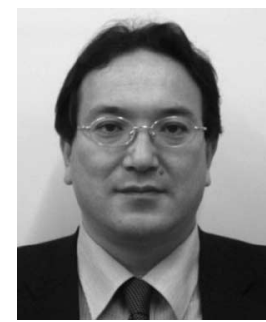

Yoichi Kawakami received the B.Sc., M.Sc., and D.Sc. degrees in electrical engineering from Osaka University, Osaka, Japan, in 1984, 1986, and 1989, respectively.

He was a Research Associate in the Department of Electrical Engineering, Kyoto University, Kyoto, Japan, where he became an Associate Professor in 1997, was promoted to a Professor in the Department of Electronic Science and Engineering in 2007, and was engaged in research on II-VI widegap semiconductors. During 1991-1992, he was a Visiting Research Fellow in the Department of Physics, Heriot-Watt University, U.K. His current research interests include spatial and temporal luminescence spectroscopy of excitons in GaN-based low-dimensional semiconductors, fabrication of new photonic devices, as well as application of solid-state lighting using white light-emitting diodes. 